\title{
Simulações Computacionais dos Processos de Manufatura Aditiva de Metais: Um Review Introdutório
}

\author{
Rodrigo Martins Farias ${ }^{1}$ (D), Louriel Oliveira Vilarinho ${ }^{1}$ (D) \\ ${ }^{1}$ Universidade Federal de Uberlândia - UFU, Faculdade de Engenharia Mecânica, Uberlândia, MG, Brasil.
}

Como citar: Farias RM, Vilarinho LO. Simulações computacionais dos processos de manufatura aditiva de metais: um review introdutório. Soldagem \& Inspeção. 2022;27:e2703. https://doi.org/10.1590/0104-9224/SI27.03

\begin{abstract}
Resumo: Os processos de Manufatura Aditiva (MA) de Metais permitem criar peças tridimensionais através da adição progressiva de finas camadas de material. Estes processos se enquadram em duas grandes categorias, denominadas Fusão em Leito de Pó e Deposição por Energia Direcionada. A compreensão física dos processos de MA pode fornecer uma visão holística sobre as sensibilidades aos parâmetros de processo. Já a modelagem computacional destes processos não só pode fornecer uma visão importante dos fenômenos físicos que levam às propriedades finais do material e à qualidade do produto, mas também os meios para explorar o espaço de projeto, visando criar produtos funcionais. Esta revisão introdutória foca na MA de materiais metálicos, particularmente nas questões de modelagem física e numérica. São apresentadas considerações sobre os diversos fenômenos físicos atuantes, métodos computacionais mais empregados, técnicas para realizar a modelagem e suas respectivas validações, demonstrando os desafios e limitações atuais, assim como as perspectivas futuras da simulação computacional aplicada aos processos de MA.
\end{abstract}

Palavras-chave: Manufatura aditiva; Simulação numérica, PBF, DED, MADA.

\section{Computational Simulations of Metal Additive Manufacturing Processes: An Introductory Review}

\begin{abstract}
Additive Manufacturing (AM) processes of metals allow creating three-dimensional parts through the progressive addition of thin layers of material. These processes fall into two major categories, called Powder Bed Fusion and Directed Energy Deposition. Physical understanding of the AM processes can provide a holistic view of the sensitivity to process parameters. Computational modeling of these processes can not only provide an important view of the physical phenomena that lead to the final properties of the material and quality of the product, but also the means to explore the design space, in order to create functional products. This introductory review focuses on the AM of metallic materials, particularly on issues of physical and numerical modeling. Considerations are presented about the various physical phenomena at work, most used computational methods, techniques to perform the modeling and their respective validations, demonstrating the current challenges and limitations, as well as the future perspectives for computer simulations applied to AM processes.
\end{abstract}

Keywords: Additive manufacturing, Numerical simulation, PBF, DED, WAAM.

\section{Introdução}

Os processos de Manufatura Aditiva (MA) de metais permitem criar peças tridimensionais (3D) adicionando progressivamente finas camadas de materiais, a partir de um modelo digital (CAD). Este processo permite a produção de peças complexas e/ou personalizadas, sem a necessidade de ferramentas dispendiosas, como punções, matrizes ou moldes de fundição, e muitas vezes reduzindo a necessidade de muitas etapas adicionais de pós-processamento (DebRoy et al., 2018). Peças detalhadas podem ser feitas em uma única etapa, sem as limitações (canais curvos p. ex.) de processos convencionais como a usinagem. Além disso, uma significativa redução na quantidade de peças de um componente pode ser realizada, visto que todo componente pode ser depositado como uma única peça, dispensando a necessidade de várias conexões. Adicionalmente, peças podem ser produzidas sob demanda, reduzindo estoques de reposição e diminuindo o tempo de espera para situações críticas, como manutenção corretiva. Por essas razões, a MA é agora amplamente aceita como uma nova maneira de projetar e produzir componentes de alto desempenho para diversas aplicações.

De acordo com a norma ISO/ASTM 52900:2015(E) (American Society for Testing and Materials, 2015), os processos MA se enquadram em duas grandes categorias: MA por Fusão em Leito de Pó (PBF - Powder Bed Fusion); e MA por Deposição por Energia Direcionada (DED - Directed Energy Deposition). Outras divisões importantes incluem a forma de distribuição do material sendo depositado (via leito de pó ou bocal de deposição) e a forma da matéria-prima (pó ou filamento/arame). Apesar 
de inúmeros processos em leito de pó (Schoinochoritis et al., 2017), a maioria dos equipamentos comercializados se restringem a fusão seletiva a laser (SLM - Selective Laser Melting) e fusão por feixe de elétrons (EBM - Eletron Beam Melting). Já para os processos $D E D$, a maior variação ocorre na fonte de calor usada para fundir o material, sendo os mais utilizados o laser (DED-L), o feixe de elétrons (DED-EB) e o arco elétrico, geralmente denominado Manufatura Aditiva por Deposição a Arco (MADA) (WAAM - Wire Arc Additive Manufacturing, ou DED-GMA).

A compreensão física do processo de MA pode fornecer um olhar abrangente sobre as sensibilidades aos parâmetros de processo (King et al., 2015). Isto é essencial na qualificação do componente depositado. Essa compreensão leva a um maior controle do processo, melhorando a probabilidade de produção de peças qualificadas. A modelagem e a simulação dos processos de MA fornecem um modo poderoso para atingir esse objetivo. Vários autores (Srivatsa, 2014) destacam as necessidades e benefícios da modelagem, sendo uma metodologia eficiente que tende a evitar a realização de um grande número de experimentos físicos.

Dado o grande número de parâmetros de processo (Megahed et al., 2016) e suas complexas interações, extensa pesquisa experimental é necessária para garantir a produção sem falhas de modelos CAD via MA, o que tende a ser dispendioso. Logo, a modelagem de processos de MA não só fornece uma visão importante dos fenômenos físicos que levam às propriedades finais do material e à qualidade do produto, mas também fornece os meios para explorar o espaço de projeto, visando criar produtos e materiais funcionais. As escalas de comprimento e tempo necessárias para modelar processos de MA e prever as características finais da peça são muito desafiadoras.

Esta revisão introdutória foca na MA de materiais metálicos, particularmente nas questões de modelagem física e numérica, voltada aos processos PBF e DED. Processos como a Sinterização Seletiva a Laser (SLS - Selective Laser Sintering), Binder Jetting, entre outros não estão dentro do escopo deste texto. São apresentadas considerações sobre fenômenos físicos atuantes, métodos computacionais mais utilizados, técnicas para fazer a modelagem e suas validações, levando em conta os desafios e limitações atuais, assim como as perspectivas futuras da simulação da MA. Espera-se que este review seja útil para entender o estado atual das tecnologias de simulação na MA, assim como algumas das necessidades de pesquisa para o avanço e expansão da MA de materiais metálicos.

\section{Fenômenos Físicos}

A modelagem baseada em física tem o potencial de expandir os horizontes sobre como os parâmetros de processo interagem, fornecendo uma base para a otimização do processo de MA. Os modelos podem prever as propriedades da peça depositada (as-built), e auxiliar na qualificação rápida de componentes plenamente funcionais. Dependendo da análise desejada, os modelos muitas vezes devem ser multifísicos, pois fenômenos térmicos, mecânicos e metalúrgicos ocorrem em paralelo, e muitas vezes são acoplados entre si. E os fenômenos também são multiescala (no comprimento e no tempo), tornando necessário dividir os modelos em modelos de micro, meso e macroescala (Vogel et al., 2013). Os fenômenos da MA envolvem desde a escala de tamanho de grão $(\mu \mathrm{m})$ até a escala do componente (alguns metros); e desde a escala de tempo de milissegundos (pulsação de uma fonte laser) até a escala de horas, necessária para fabricar peças de grandes dimensões.

\subsection{Fenômenos térmicos}

Os processos PBF e DED são razoavelmente similares, possuindo alguns fenômenos de natureza térmica em comum: condução de calor através do material que foi depositado; transferência de calor da superfície da peça depositada para o ambiente através de convecção e radiação; convecção e advecção do metal líquido na poça de fusão; grande intensidade de calor (laser, feixe de elétrons, arco elétrico) localizado em uma região muito pequena; evaporação metálica a altas temperaturas na poça de fusão, entre outros fenômenos. Pode-se citar como diferença entre os processos o fato de que a transferência de calor das laterais da peça se dá para o ambiente no caso do $D E D$, enquanto no processo PBF esse calor é transferido para o pó não fundido que circunda a peça sendo depositada. Outra grande diferença está na escala desses fenômenos. Visto que peças feitas pelos processos $P B F$ tendem a ser bem menores quando comparadas as fabricadas por processos $D E D$, estes processos geralmente possuem poças de fusão pequenas, que tendem a gerar taxas de resfriamento muito elevadas.

Na Figura 1a (Shi et al., 2016) temos os fenômenos térmicos relacionados aos processos em leito de pó, neste caso, o processo SLM. Na Figura 1b (Liskevych et al., 2013) temos os possíveis fenômenos relacionados aos processos de energia direcionada. Neste caso, os fenômenos oriundos da soldagem são os mesmo que surgem no processo MADA (WAAM) por exemplo.

\subsection{Fenômenos mecânicos}

Uma consequência inerente da deposição de metal líquido em um substrato relativamente mais frio, ou em camadas previamente depositadas, é o gradiente de temperatura acentuado, que leva a deformação térmica e consequentes tensões residuais (DebRoy et al., 2018). Estas podem levar à distorção da peça, perda de tolerância geométrica, delaminação das camadas durante a deposição, bem como deterioração do desempenho da peça fabricada. Um conhecimento adequado da evolução das tensões térmicas durante a MA é essencial para compreender e controlar/mitigar estes problemas. Por exemplo, para processos de MA em leito de pó, a distorção da peça pode ser grande o suficiente para evitar que o sistema de nivelamento espalhe a fina camada de pó na área de deposição. Uma compreensão das tensões térmicas também pode auxiliar a otimizar a colocação de estruturas de suporte a fim de minimizar as distorções geradas pelo processo. 
(a)

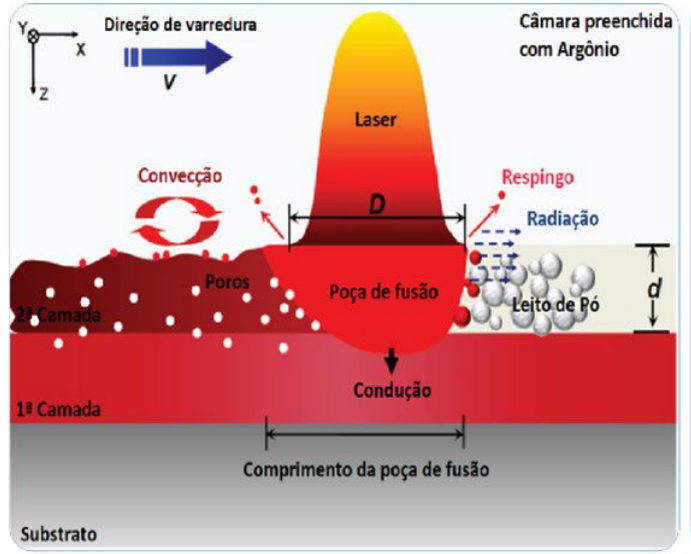

(b)

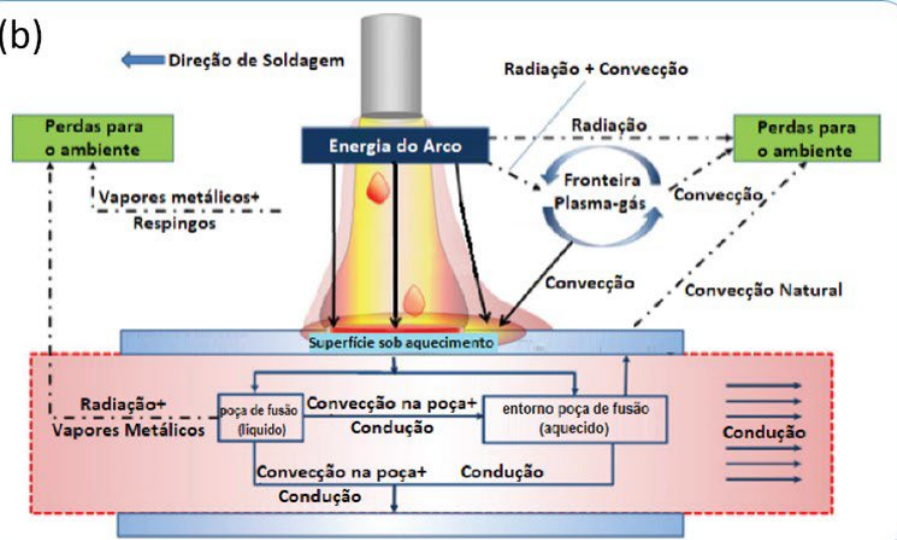

Figura 1. Processo SLM (Shi et al., 2016) e processo soldagem (Liskevych et al., 2013), equivalente ao MADA.

(Figuras traduzidas e adaptadas pelo autor).

Na MA, tem-se três principais aspectos físicos responsáveis pelo surgimento de distorções e tensões residuais (Goldak e Akhlaghi, 2006): gradientes elevados de temperatura devido ao aquecimento e resfriamento localizado, causado pela fonte de calor móvel; expansão e contração térmica do material devido aos inúmeros ciclos térmicos; e compatibilidade de deformações (distribuição desigual de deformações inelásticas), equilíbrio das forças e comportamento constitutivo da relação tensão-deformação.

Em relação as deformações, a seguinte divisão destas é abordada: deformação elástica; plástica; térmica, deformação por transformação de fases, e por fluência. As duas últimas costumam ser desconsideradas, no entanto, alguns trabalhos (Pellereau et al., 2013) demonstram que para alguns tipos de aço as mudanças de fase sólida levam a grandes diferenças nas tensões previstas, e devem ser levadas em consideração.

\subsection{Fenômenos Metalúrgicos}

Diversos aspectos metalúrgicos podem ser observados na MA, e modelados computacionalmente. Questões da solidificação do metal depositado como nucleação e crescimento de grãos; orientação, tamanho e morfologia das estruturas granulares (Wei et al., 2016); textura, transformações de fase e composição química final da peça, apenas para citar alguns tópicos (Kou, 2003). O estudo destas questões é importante, pois influem diretamente nas propriedades do componente manufaturado, como resistência mecânica, à fadiga e ao impacto, dureza, assim como comportamento sob corrosão.

\section{Métodos Computacionais}

Os métodos numéricos podem ser classificados em métodos baseados em malha e métodos livres de malha (Parandoush e Hossain, 2014). O Método dos Elementos Finitos (FEM - Finite Element Method) pertence à primeira classe. Nele, o domínio sob estudo é discretizado em um número finito de elementos formando uma malha, reduzindo assim o problema a um número finito de incógnitas. Devido as suas características, o FEM tornou-se a técnica mais comum para prever a ciclos térmicos, distorções e tensões residuais de peças produzidas por processos de MA. Outros métodos, como Método dos Volumes Finitos (Gu e Li, 2019) e o Método Lattice Boltzmann são utilizados para estudar a fluidodinâmica na poça de fusão, assim como outros fenômenos físicos mais complexos. O Método dos Elementos Discretos (DEM - Discrete Element Method) tem sido uma escolha interessante para modelar o comportamento do leito de pó (Ganeriwala e Zohdi, 2016). Para os fenômenos metalúrgicos, métodos como CALPHAD (Smith et al., 2016), Monte Carlo, Cellular Automata (Zinoviev et al., 2016) e Phase Field (Keller et al., 2017) são alguns dos mais utilizados.

\section{Modelagem}

A seguir são apresentados os diferentes tipos de modelagens aplicados aos processos de MA.

\subsection{Modelagem térmica}

Para modelagem dos fenômenos térmicos, basicamente são utilizados três modelos (Gan et al., 2019), em ordem crescente de complexidade:

1. Modelo de Condução de Calor: soluciona apenas a equação da difusão de calor, considerando condições de contorno de convecção e radiação, e entrada de calor do processo através de fluxos prescritos.

2. Modelo Termofluidodinâmico: considera a movimentação do metal na poça de fusão, dirigida principalmente pelo efeito Marangoni. Além da conservação de energia, são utilizadas as equações de conservação de massa e momentum. 
3. Modelo Termofluidodinâmico com vaporização: a perda de material e calor através da vaporização do metal é acrescentada as formulações do modelo anterior.

Os modelos tipo 1 são os mais utilizados em simulações termomecânicas da MA (Smith et al., 2016) (Luo e Zhao, 2018), onde é de interesse prever os ciclos térmicos e taxas de resfriamento, e a partir destes, distorções e tensões residuais no componente depositado. É o de menor tempo computacional dentre os três modelos, muito usado para modelagens na escala de tamanho da peça. Os modelos do tipo 2 geralmente são utilizados em escala meso, para simular apenas um cordão de uma camada. Geralmente são usados para determinar o comportamento da poça de fusão e sua variação com os parâmetros do processo, a fim de assessorar a seleção de parâmetros dos experimentos (Xia et al., 2017). Modelos do tipo 3 e além são os que exigem maior tempo computacional, e também são usados na escala meso, para por exemplo determinar o surgimento de porosidade, ou possibilidade de formação do modo keyhole em processos com fonte laser (PBF e DED). Informações obtidas destes três tipos de modelos são necessárias para simular os fenômenos mecânicos e metalúrgicos, e sua escolha depende dos objetivos gerais da modelagem sendo realizada (acurácia, tempo computacional, validações disponíveis, etc.).

\subsection{Modelagem mecânica}

Distorções e tensões residuais na MA são fenômenos que ocorrem em toda a peça. Simular toda a construção da peça no substrato é essencial para calcular com acurácia essas grandezas. Para modelos em escala macro (ordem de tamanho da peça), o modelo mecânico de MA não é adequado para simular diretamente a adição de material (p. ex., partículas de pó fundindo na poça de fusão). Em vez disso, depende da adição de material por meio da malha. Logo, uma malha para toda a peça é criada seguindo o caminho de deposição, onde a forma e tamanho do cordão devem ser conhecidos a priori. O cordão geralmente tem seção transversal retangular para facilitar o preenchimento do volume da peça por passes individuais.

Conforme apresentado no Item 1, existem vários tipos de processos de MA, que exigem diferentes procedimentos de modelagem. A abordagem da modelagem mecânica para processos DED é essencialmente a mesma da soldagem por fusão. 0 domínio da solução é dividido para incluir apenas o substrato sólido e as camadas depositadas, e nenhum pó ou arame (Martukanitz et al., 2014). Por outro lado, a presença da camada de pó pré-colocada dos processos PBF requer um tratamento especial, uma vez que as partículas de pó têm uma resposta térmica e mecânica diferente do metal sólido consolidado (King et al., 2015). Apesar da necessidade de manusear o material em pó, a abordagem padrão de resolução de tensões e deformações permanece a mesma para os processos PBF e DED.

Enquanto a etapa térmica dos processos MA envolve uma análise térmica transiente não linear, a modelagem da etapa mecânica geralmente é realizada via análise mecânica elastoplástica quasi-estática. Após a obtenção do campo de temperaturas a partir da análise térmica, a análise mecânica é executada para prever as tensões térmicas e a deformação. O campo de temperatura é aplicado como uma carga térmica na análise mecânica. Esta análise termomecânica pode ser realizada de forma acoplada ou desacoplada (Schoinochoritis et al., 2017). Para a análise acoplada, a expansão térmica irá influenciar as propriedades mecânicas, enquanto a deformação plástica induzida pela expansão térmica irá influenciar as propriedades térmicas por sua vez. Portanto, as análises térmicas e mecânicas devem ser realizadas simultaneamente para cada passo de tempo. Para a análise desacoplada, o calor de atrito induzido pela deformação plástica é muito menor em comparação com a energia térmica do processo, o que significa que a interação entre o campo de temperatura e o campo de tensão pode ser desconsiderada. Portanto, as análises térmicas e mecânicas podem ser realizadas acopladas sequencialmente. Na maioria dos casos existentes (Cheng et al., 2016) (Farias et al., 2017) (Luo e Zhao, 2018), o método de análise termomecânica fracamente acoplado é preferido devido ao menor custo computacional.

\subsection{Modelagem metalúrgica}

A partir das taxas de resfriamento e gradiente térmico obtidos a partir do modelo térmico, diversas grandezas metalúrgicas podem ser calculadas (Gan et al., 2019). O espaçamento dos braços primários das dendritas pode ser previsto usando o modelo de Kurz-Fisher. A segregação de elementos de liga pode ser avaliada com base no modelo Scheil-Gulliver com hipótese de equilíbrio termodinâmico ou com solidificação sem equilíbrio para ligas com múltiplos componentes (Liang et al., 2016).

Para prever estrutura, tamanho e morfologia de grãos, existem métodos estocásticos e determinísticos que simulam a nucleação e crescimento de grãos. Três métodos são os mais utilizados atualmente (em ordem de complexidade):

1. Kinetic Monte Carlo $(K M C)$ : método estocástico, onde os grãos individuais são representados, associando cada local de rede a um grão, e utiliza um modelo de Potts para simular a solidificação e o crescimento dos grãos, modificando os valores das células em cada etapa durante a aplicação do método Monte Carlo, para reduzir as energias de superfície entre grãos diferentes. As morfologias finais dos grãos tendem a estar de acordo com os resultados experimentais observados (Rodgers et al., 2017), no entanto, a abordagem KMC carece de um modelo para capturar com precisão a velocidade de solidificação de grãos individuais.

2. Cellular Automata (CA) (Lian et al., 2018): método estocástico-determinístico. No método CA, os grãos são rastreados em uma grade discreta de células na qual células individuais podem ser atribuídas a um estado particular (Rappaz e Gandin, 
1993), semelhante à descrição KMC. A nucleação de novos grãos é modelada estocasticamente dependendo do subresfriamento local das células no estado líquido, enquanto os grãos crescem (efetivamente "capturando" células vizinhas) de acordo com um modelo determinístico de crescimento das dendritas, baseado na velocidade da ponta da dendrita, que por sua vez depende do sub-resfriamento local de cada grão. Como os grãos crescem baseados em uma lei física, os resultados deste método tendem a ser melhores que os do KMC.

3. Phase Field (Zhang et al., 2020): método determinístico, em que uma variável de campo varia suavemente ao longo de uma região de interface difusa para demarcar diferentes fases (Boettinger et al., 2002), têm sido usado para modelar o crescimento de estruturas dendríticas em materiais puros e ligas. É uma abordagem valiosa para entender os detalhes finos de uma estrutura de solidificação. No entanto, a pequena escala de comprimento e o pequeno número de grãos que podem ser obtidos usando esse método torna difícil prever microestruturas em tamanhos que podem ser ligados ao desempenho da peça depositada.

\subsection{Modelo de material}

Um modelo de material eficaz, que considere a variação das propriedades durante os sucessivos ciclos de aquecimento e resfriamento de material durante o processo de MA é fundamental (Fu e Guo, 2014). Como a temperatura do material varia desde a temperatura ambiente $\left(\sim 20^{\circ} \mathrm{C}\right)$ até a temperatura de fusão dos metais $\left(\sim 630^{\circ} \mathrm{C}\right.$ para ligas de alumínio, $\sim 1400{ }^{\circ} \mathrm{C}$ e acima para ligas de aço e outros metais), a maioria das propriedades muda drasticamente. E mudanças de fase também podem causar variações nestas propriedades. Esta dependência da temperatura é um dos fatores mais importantes na análise de tensões térmicas. Uma definição adequada das propriedades é necessária para um modelo com boa acurácia. Além disso, as propriedades e comportamentos das partículas de metal, para os processos $P B F$, são completamente diferentes do metal sólido. Logo, tanto os materiais em pó quanto os sólidos podem (e devem) ser especificados como dependentes da temperatura.

As propriedades termofísicas incluem massa específica, condutividade térmica, calor específico e entalpia do material. $\mathrm{A}$ mudança de fase sólida para líquida deve ser considerada através do calor latente. Em modelos que usam o calor específico, a mudança de fase é contemplada alterando-se esta propriedade na faixa de fusão do material. Já com o uso da entalpia, a mudança de fase faz parte da curva desta propriedade, e o calor latente já está incluído. Modelos que usam a entalpia possuem uma melhor acurácia e maior estabilidade numérica (Yan et al., 2018) quando comparados aos que usam o calor especifico. Em processos $P B F$, é adequado considerar a condutividade térmica do pó metálico solto até cerca de 100 vezes menor que a do material sólido (Gu e Yuan, 2015). Em modelos termomecânicos, propriedades como módulo de elasticidade, tensão de escoamento e coeficiente de expansão térmica também devem variar com a temperatura.

\subsection{Fontes de calor}

A modelagem da fonte de calor é o aspecto mais fundamental da análise computacional dos processos de MA. Toda energia que entra no sistema o faz através da fonte de calor. A maioria dos modelos atuais de fontes de calor para MA são os mesmos utilizados nas simulações de soldagem, visto que a física fundamental do processo é a mesma. Além dos modelos de fonte de calor pontuais ou em linha, geralmente usados em modelos analíticos (Ríos et al., 2018), fontes de calor de superfície com distribuição gaussiana e de volume permanecem as mais populares até o momento. Os modelos tradicionais são simples e eficientes, mas não são capazes de capturar algumas das informações mais detalhadas relacionadas aos processos físicos complexos observados na MA. Al Hamahmy e Deiab (2020) e Luo e Zhao (2018) apresentam uma extensa lista de fontes de calor para uso em simulações de MA.

Conforme apresentado, existem diferentes fontes primárias de energia usadas em aplicações de MA. Fontes laser, de feixes de elétrons e a arco elétrico são caracterizados por diferentes fenômenos físicos. Logo, diferentes tipos de entrada de energia, com diferentes fenômenos físicos, necessitam de modelos de fonte específicos. Portanto, é desejável e útil que novos modelos de fonte de calor sejam desenvolvidos de acordo com os mecanismos físicos reais particulares de cada processo. Por exemplo, o pó usado em processos $P B F$ tem efeitos significativos na distribuição da energia e na condução térmica. A interação entre o feixe de elétrons/laser e o pó metálico resulta em distribuições de energia que diferem muito daquela observada na soldagem, enquanto processos de MA baseados em arco elétrico se aproximam mais dos seus equivalentes em soldagem. Ou seja, o modelo da fonte de calor deve ser selecionado com base no tipo e estado do material sendo processado.

\subsection{Modelagem da deposição de material}

Dependendo do referencial escolhido (Euleriano ou Lagrangiano), a representação da deposição de metal possui diferentes implementações. Os métodos a seguir foram desenvolvidos e melhorados para modelos da soldagem originalmente. Quando as simulações transientes são realizadas dentro de uma abordagem Euleriana, métodos de rastreamento de interface, como Volume de Fluido (VOF - Volume of Fluid) ou Level Set Method são usados. Apesar de ferramentas numéricas avançadas, os parâmetros numéricos inerentes ao processo tornam esta abordagem muito complexa e de alto custo computacional para modelagem de peças grandes e complexas via MA. Em comparação, o referencial Lagrangiano permite fácil manuseio de uma 
técnica de ativação/desativação de elementos, nomeada conforme o código FEM usado: método de ativação de elementos no Sysweld $^{\circledR}$ e MSC Marc $^{\circledR}$ (Neugebauer et al., 2014), técnica de nascimento de elementos com Abaqus ${ }^{\circledR}$ (Ding et al., 2011) e ANSYS $^{\circledR}$ (Mayer et al., 2020). Esses métodos podem ser classificados em duas categorias, o método dos elementos "silenciosos" ou método dos elementos "inativos".

O método do elemento "silencioso" é baseado na existência inicial de todos os elementos do modelo. As propriedades dos elementos "silenciosos" diferem daquelas dos elementos "ativos", onde fatores de escala (multiplicadores) são usados para a condutividade e o calor específico (deixando-os próximo de zero). O método do elemento "inativo" remove os elementos que representam o metal a ser depositado desde o cálculo até sua ativação. Michaleris (2014) comparou ambas as técnicas em termos de acurácia e tempo computacional para a análise térmica, propondo em seu trabalho um método de elemento híbrido "silencioso"/"inativo", em que os elementos da camada depositada atual seriam alternados para "silenciosos" e os de outras camadas seriam alternados para "inativos". Qualquer que seja o método escolhido, a acurácia das tensões térmicas é atendida e o tempo computacional é diminuído.

\section{Validações Experimentais}

Algum nível de abstração sempre é utilizado ao criar as hipóteses dos modelos de simulação, para que o tempo computacional seja viável. Assim, a validação do modelo de simulação através da comparação com resultados experimentais é recomendada e obrigatória. Para modelos térmicos, os ciclos térmicos da peça durante a fabricação podem ser registrados experimentalmente com instrumentos como termopares, pirômetros e câmeras termográficas (Schoinochoritis et al., 2017). Enquanto o termopar mede a temperatura apenas no ponto onde está instalado, câmeras termográficas (Silva et al., 2020) podem fornecer uma imagem de distribuição de temperatura, provendo informações em uma área maior. No entanto, estas exigem maiores cuidados de calibração.

No campo da mecânica, as tensões residuais em MA são altamente não uniformes e variam no espaço e no tempo durante a deposição. A medição experimental de tensões residuais é normalmente limitada a alguns pontos discretos ou contornos em planos selecionados da peça após a fabricação ser concluída. Diversas técnicas tem sido usadas para medir tensões residuais após a deposição, sendo as mesmas adaptadas da soldagem. Várias revisões sobre medição de tensão residual estão disponíveis na literatura para técnicas gerais e aplicadas a MA. Tensões residuais em peças fabricadas por MA podem ser medidas indiretamente por micro-indentação Vickers, e diretamente por métodos como furo cego, strain gauges, raios- $X$ e difração de nêutrons. A técnica do furo cego geralmente sofre de baixa resolução e maiores incertezas. Técnicas baseadas na difração de raios-X (Liu et al., 2016), embora superiores aos métodos de perfuração, penetram pouco na superfície para a medição de tensões residuais. Em contraste, a difração de nêutrons penetra a uma profundidade maior na maioria dos materiais metálicos e é considerada a técnica adequada para medições mais confiáveis em peças de uso crítico. DebRoy et al. (2018) apresenta uma extensa tabela-resumo com estudos da literatura sobre medição de tensões residuais e distorção, para diferentes materiais e processos de MA de metais.

Para avaliações metalúrgicas, as técnicas tradicionais de caracterização microestrutural também podem ser aplicadas as peças feitas por MA. No entanto, devido aos atributos únicos da MA, dois pontos principais requerem atenção especial: densidade das ligas fabricadas, ou seja, vazios/porosidade, especialmente para os processos PBF; e anisotropia microestrutural, ou seja, distribuição de defeitos, inclusões, orientação e morfologia de grãos, atribuídos aos ciclos de reaquecimento (Smith et al., 2016). Técnicas como espectroscopia EDX (Energy-Dispersive X-ray), EBSD (Electron Backscatter Diffraction) e microscopia de Força Atômica (AFM-Atomic Force Microscopy) são utilizadas para validações de modelagens metalúrgicas.

\section{Desafios e Limitações}

Alguns pontos podem ser destacados sobre técnicas utilizadas atualmente, ressaltando suas vantagens e limitações. $\mathrm{O}$ uso de técnicas de malhas adaptativas apresenta a vantagem da redução dos tempos computacionais (Denlinger e Michaleris, 2016), no entanto, sua implementação para geometrias intrincadas é complexa. Simulações multifísicas ou com acoplamentos entre os diferentes fenômenos tendem a trazer uma maior fidelidade na representação do processo de MA, mas o custo computacional cresce exponencialmente com a adição de fenômenos (Cadiou et al., 2020). O uso de técnicas de computação paralela, usando múltiplos processadores (CPUs/GPUs) diminui significativamente os tempos computacionais, entretanto, nem todos tipos de modelos podem ser facilmente paralelizáveis (Lian et al., 2018), além ainda do custo adicional de tais equipamentos. $\mathrm{O}$ uso de modelos de inteligência artificial e aprendizado de máquinas (Machine Learning) permite resultados instantâneos e identificação de padrões no processo (Stathatos e Vosniakos, 2019), mas geralmente é necessário grande número de experimentos para alimentar estes modelos com dados suficientes para que tenham boa acurácia.

\section{Perspectivas Futuras}

Um ponto unânime entre os estudiosos da modelagem da Manufatura Aditiva de Metais é que o tempo computacional continua sendo o maior desafio para o futuro, principalmente para uma maior adoção deste tipo de simulação na indústria. A 
criação de bancos de dados com propriedades de materiais confiáveis, necessárias para a modelagem, também é outro ponto a se destacar. Com a evolução dos equipamentos de simulação e capacidade computacional instalada, também esperasse uma maior adoção de modelagens multifísicas, trazendo maior fidelidade aos resultados obtidos e unindo as diferentes escalas de tempo e comprimento presentes na MA. Novas e aprimoradas técnicas de medição também são necessárias para uma melhor validação dos modelos numéricos. E uma maior qualificação de engenheiros de simulação para modelagem dos fenômenos da Manufatura Aditiva é considerada obrigatória para a expansão do uso destes processos, tanto na pesquisa quanto nas aplicações industriais.

\section{Agradecimentos}

Este estudo faz parte dos projetos financiados pela Petrobras S.A. (projetos 2019/00123-1 e 2019/00124-8) e foi apoiado pela Fundação de Amparo à Pesquisa do Estado de Minas Gerais (Fapemig), Conselho Nacional de Desenvolvimento Científico e Tecnológico (CNPq), Coordenação de Aperfeiçoamento de Pessoal de Nível Superior (Capes) e Universidade Federal de Uberlândia (UFU).

\section{Referências}

Al Hamahmy MI, Deiab I. Review and analysis of heat source models for additive manufacturing. International Journal of Advanced Manufacturing Technology. 2020;106:1223-1238. http://dx.doi.org/10.1007/s00170-019-04371-0.

American Society for Testing and Materials. ISO/ASTM 52900:2015 - Standard terminology for additive manufacturing - General Principles Terminology. West Conshohocken: ASTM International; 2015.

Boettinger WJ, Warren JA, Beckermann C, Karma A. Phase-field simulation of solidification. Annual Review of Materials Research. 2002;32(1):163-194. http://dx.doi.org/10.1146/annurev.matsci.32.101901.155803.

Cadiou S, Courtois M, Carin M, Berckmans W. 3D heat transfer, fluid flow and electromagnetic model for cold metal transfer wire arc additive manufacturing (Cmt-Waam). Additive Manufacturing. 2020;36:101541.

Cheng B, Shrestha S, Chou K. Stress and deformation evaluations of scanning strategy effect in selective laser melting. Additive Manufacturing. 2016;12:240-251. http://dx.doi.org/10.1016/j.addma.2016.05.007.

DebRoy T, Wei HL, Zuback JS, Mukherjee T, Elmer JW, Milewski JO, et al. Additive manufacturing of metallic components - process, structure and properties. Progress in Materials Science. 2018;92:112-224. http://dx.doi.org/10.1016/j.pmatsci.2017.10.001.

Denlinger ER, Michaleris P. Effect of stress relaxation on distortion in additive manufacturing process modeling. Additive Manufacturing. 2016;12:51-59. http://dx.doi.org/10.1016/j.addma.2016.06.011.

Ding J, Colegrove P, Mehnen J, Ganguly S, Almeida PS, Wang F, et al. Thermo-mechanical analysis of Wire and Arc Additive Layer Manufacturing process on large multi-layer parts. Computational Materials Science. 2011;50(12):3315-3322. http://dx.doi.org/10.1016/j.commatsci.2011.06.023.

Farias RM, Teixeira PRF, Araújo DB. Thermo-mechanical analysis of the MIG/MAG multi-pass welding process on AISI 304L stainless steel plates. Journal of the Brazilian Society of Mechanical Sciences and Engineering. 2017;39(4):1245-1258. http://dx.doi.org/10.1007/s40430-016-0574-y.

Fu CH, Guo YB. Three-dimensional temperature gradient mechanism in selective laser melting of Ti-6Al-4V. Journal of Manufacturing Science and Engineering. 2014;136(6):061004. http://dx.doi.org/10.1115/1.4028539.

Gan Z, Lian Y, Lin SE, Jones KK, Liu WK, Wagner GJ. Benchmark study of thermal behavior, surface topography, and dendritic microstructure in selective laser melting of inconel 625. Integrating Materials and Manufacturing Innovation. 2019;8(2):178-193. http://dx.doi.org/10.1007/s40192-019-00130-x.

Ganeriwala R, Zohdi TI. A coupled discrete element-finite difference model of selective laser sintering. Granular Matter. 2016;18(2):21. http://dx.doi.org/10.1007/s10035-016-0626-0.

Goldak JA, Akhlaghi M. Computational welding mechanics. 1. ed. Boston: Springer; 2006.

Gu D, Yuan P. Thermal evolution behavior and fluid dynamics during laser additive manufacturing of Al-based nanocomposites: underlying role of reinforcement weight fraction. Journal of Applied Physics. 2015;118(23):233109. http://dx.doi.org/10.1063/1.4937905.

$\mathrm{Gu} \mathrm{H,} \mathrm{Li} \mathrm{L.} \mathrm{Computational} \mathrm{fluid} \mathrm{dynamic} \mathrm{simulation} \mathrm{of} \mathrm{gravity} \mathrm{and} \mathrm{pressure} \mathrm{effects} \mathrm{in} \mathrm{laser} \mathrm{metal} \mathrm{deposition} \mathrm{for} \mathrm{potential} \mathrm{additive}$ manufacturing in space. International Journal of Heat and Mass Transfer. 2019;140:51-65. http://dx.doi.org/10.1016/j.ijheatmasstransfer.2019.05.081.

Liskevych O, Quintino L, Vilarinho LO, Scotti A. Intrinsic errors on cryogenic calorimetry applied to arc welding. Welding in the World. 2013;57:349-357. http://dx.doi.org/10.1007/s40194-013-0035-5.

Keller T, Lindwall G, Ghosh S, Ma L, Lane B, Zhang F, et al. Application of finite element, phase-field, and CALPHAD-based methods to additive manufacturing of Ni-based superalloys. Acta Materialia. 2017;139:244-253. http://dx.doi.org/10.1016/j.actamat.2017.05.003. PMid:29230094.

King WE, Anderson AT, Ferencz RM, Hodge NE, Kamath C, Khairallah SA, et al. Laser powder bed fusion additive manufacturing of metals; physics, computational, and materials challenges. Applied Physics Reviews. 2015;2(4):041304. http://dx.doi.org/10.1063/1.4937809. 
Kou S. Welding metallurgy. 2. ed. Hoboken: John Wiley \& Sons; 2003.

Lian Y, Lin S, Yan W, Liu WK, Wagner GJ. A parallelized three-dimensional cellular automaton model for grain growth during additive manufacturing. Computational Mechanics. 2018;61(5):543-558. http://dx.doi.org/10.1007/s00466-017-1535-8.

Liang YJ, Cheng X, Wang HM. A new microsegregation model for rapid solidification multicomponent alloys and its application to singlecrystal nickel-base superalloys of laser rapid directional solidification. Acta Materialia. 2016;118:17-27. http://dx.doi.org/10.1016/j.actamat.2016.07.008.

Liu Y, Yang Y, Wang D. A study on the residual stress during selective laser melting (SLM) of metallic powder. International Journal of Advanced Manufacturing Technology. 2016;87:647-656. http://dx.doi.org/10.1007/s00170-016-8466-y.

Luo Z, Zhao Y. A survey of finite element analysis of temperature and thermal stress fields in powder bed fusion Additive Manufacturing. Additive Manufacturing. 2018;21:318-332. http://dx.doi.org/10.1016/j.addma.2018.03.022.

Martukanitz R, Michaleris P, Palmer T, Debroy T, Liu ZK, Otis R, et al. Toward an integrated computational system for describing the additive manufacturing process for metallic materials. Additive Manufacturing. 2014;1-4:52-63. http://dx.doi.org/10.1016/j.addma.2014.09.002

Mayer T, Brändle G, Schönenberger A, Eberlein R. Simulation and validation of residual deformations in additive manufacturing of metal parts. Heliyon. 2020;6(5):e03987. http://dx.doi.org/10.1016/j.heliyon.2020.e03987. PMid:32478189.

Megahed M, Mindt H-W, N'Dri N, Duan H, Desmaison O. Metal additive-manufacturing process and residual stress modeling. Integrating Materials and Manufacturing Innovation. 2016;5(1):61-93. http://dx.doi.org/10.1186/s40192-016-0047-2.

Michaleris P. Modeling metal deposition in heat transfer analyses of additive manufacturing processes. Finite Elements in Analysis and Design. 2014;86:51-60. http://dx.doi.org/10.1016/j.finel.2014.04.003.

Neugebauer F, Keller N, Ploshikhin V, Feuerhahn F, Köhler H. Multi scale FEM simulation for distortion calculation in additive manufacturing of hardening stainless steel. In Bremer Institut für Angewandte Strahltechnik. Proceedings of the international workshop on thermal forming and welding distortion; 2014 April 09-10; Bremen. Bremen: BIAS-Verl; 2014.

Parandoush P, Hossain A. A review of modeling and simulation of laser beam machining. International Journal of Machine Tools \& Manufacture. 2014;85:135-145. http://dx.doi.org/10.1016/j.ijmachtools.2014.05.008.

Pellereau B, Hurrell P, Gill C, Francis J, Bate S. Phase transformation properties sensitivity study in a ferritic groove weld. In American Society of Mechanical Engineers. Proceedings of the ASME pressure vessels and piping conference. Vol 6b: Materials and Fabrication; 2013 July 14-18; Paris. New York: ASME; 2013. http://dx.doi.org/10.1115/PVP2013-98021.

Rappaz M, Gandin CA. Probabilistic modelling of microstructure formation in solidification processes. Acta Metallurgica et Materialia. 1993;41(2):345-360. http://dx.doi.org/10.1016/0956-7151(93)90065-Z.

Ríos S, Colegrove PA, Martina F, Williams SW. Analytical process model for wire+ arc additive manufacturing. Additive Manufacturing. 2018;21:651-657. http://dx.doi.org/10.1016/j.addma.2018.04.003.

Rodgers TM, Madison JD, Tikare V. Simulation of metal additive manufacturing microstructures using kinetic Monte Carlo. Computational Materials Science. 2017;135:78-89. http://dx.doi.org/10.1016/j.commatsci.2017.03.053.

Schoinochoritis B, Chantzis D, Salonitis K. Simulation of metallic powder bed additive manufacturing processes with the finite element method: a critical review. Proceedings of the Institution of Mechanical Engineers. Part B, Journal of Engineering Manufacture. 2017;231(1):96-117. http://dx.doi.org/10.1177/0954405414567522.

Shi Q, Gu D, Xia M, Cao S, Rong T. Effects of laser processing parameters on thermal behavior and melting/solidification mechanism during selective laser melting of TiC/Inconel 718 composites. Optics \& Laser Technology. 2016;84:9-22. http://dx.doi.org/10.1016/j.optlastec.2016.04.009.

Silva RHG, Rocha PCJ, Rodrigues MB, Pereira M, Galeazzi D. Analysis of interlayer idle time as a temperature control technique in additive manufacturing of thick walls by means of CMT and CMT pulse welding processes. Soldagem e Inspeção. 2020;25:e2501. http://dx.doi.org/10.1590/0104-9224/si25.01.

Smith J, Xiong W, Yan W, Lin S, Cheng P, Kafka OL, et al. Linking process, structure, property, and performance for metal-based additive manufacturing: computational approaches with experimental support. Computational Mechanics. 2016;57(4):583-610. http://dx.doi.org/10.1007/s00466-015-1240-4.

Srivatsa S. 2014. Additive Manufacturing (AM) design and simulation tools. Study of Air Force Research Laboratory. Ohio: Wright-Patterson Air Force Base.

Stathatos E, Vosniakos GC. Real-time simulation for long paths in laser-based additive manufacturing: a machine learning approach. International Journal of Advanced Manufacturing Technology. 2019;104:1967-1984. http://dx.doi.org/10.1007/s00170-019-04004-6.

Vogel M, Khan M, Ibara-Medina J, Pinkerton A, N'Dri N, Megahed M. A coupled approach to weld pool, phase and residual stress modeling of laser direct metal deposition (LDMD) processes. In 2nd World Congress on Integrated Computational Materials Engineering; Salt Lake City. Hoboken: John Wiley \& Sons Inc. 2013. p. 231-236. http://dx.doi.org/10.1002/9781118767061.ch37. 
Wei HL, Elmer JW, Debroy T. Origin of grain orientation during solidification of an aluminum alloy. Acta Materialia. 2016;115:123-131. http://dx.doi.org/10.1016/j.actamat.2016.05.057.

Xia M, Gu D, Yu G, Dai D, Chen H, Shi Q. Porosity evolution and its thermodynamic mechanism of randomly packed powder-bed during selective laser melting of Inconel 718 alloy. International Journal of Machine Tools \& Manufacture. 2017;116:96-106. http://dx.doi.org/10.1016/j.ijmachtools.2017.01.005.

Yan Z, Liu W, Tang Z, Liu X, Zhang N, Li M, et al. Review on thermal analysis in laser-based additive manufacturing. Optics \& Laser Technology. 2018;106:427-441. http://dx.doi.org/10.1016/j.optlastec.2018.04.034.

Zhang Z, Yao XX, Ge P. Phase-field-model-based analysis of the effects of powder particle on porosities and densities in selective laser sintering additive manufacturing. International Journal of Mechanical Sciences. 2020;166:105230. http://dx.doi.org/10.1016/j.ijmecsci.2019.105230.

Zinoviev A, Zinovieva O, Ploshikhin V, Romanova V, Balokhonov R. Evolution of grain structure during laser additive manufacturing. Simulation by a cellular automata method. Materials \& Design. 2016;106:321-329. http://dx.doi.org/10.1016/j.matdes.2016.05.125. 